\title{
Polarization Dependence of the Bremsstrahlung Cross- Section in the Scattering of Electrons by Nuclei with Electromagnetic Multipole Moments
}

\author{
S. A. Al-Khateeb \\ Department of Mathematics, Faculty of Science for Girls, \\ King Abdulaziz University, Jeddah, Saaudi Arabi
}

\begin{abstract}
The present work deals with one of the most important problem in the quantum electrodynamics, 1.e the problem of bremsstrahlung process due to the interaction between lepton (electron) and the field of nuclei having electromagnetic multipole distributions. The detaild calculations for the cross-sections and the angular and energy distributions of the process are given in the first Born approximation of the quantum perturbation theory in two cases: With neglecting the polarizations of the photon and the electron, and with studying these polarizations. Applying the obtained formulae to some nuclei, the light nucleus $\left(B e_{4}^{9}\right)$ and the heavier one $\left(A l_{13}^{27}\right)$, to showing the effect of both circular photon polarization and longitudinal electron polarization on the bremsstahlung cross-section, and comparing the results obtained for the two nuclei graphically.
\end{abstract}

\section{I-Introduction}

The Study of the bremsstrahlung process was firstly done by Bethe and Heitler $^{[1]}$ in 1934, and many works about this process could have appeared in the period (1934-1954) $)^{[2-4]}$. In these works, the polarizations of the photons of bremsstrahlung and of the electrons were not studied because of the complicity of the calculations in this case.The circular polarization of bremsstrahlung photons due to the collision of longitudinally polarized electrons with nuclei, was firstly investigated by Grodzins $^{[5]}$ in 1959. At the same time, Olsen and Maximon ${ }^{[6]}$ studied the circular polarization of the bremsstrahlung photons experimentally. Motz 
and Placious $^{[7]}$ in 1960 , studied the linear polarization of the bremsstrahlung photons, and in 1962, Bisi and others ${ }^{[8]}$ made some measurements about the dependence of the degree of circular polarization of bremsstrahlung on the electric and magnetic field and on the atomic number of the target nuclei. The intensity of bremsstrahlung due to the interaction of electric and magnetic interactions between electrons and target nucleus, had been calculated by Ginsberg and Pratt ${ }^{[9]}$ in 1964, and in the next year, Mork and Olsen ${ }^{[10]}$ investigated the polarization effect on bremsstrahlung, and showed that the study of polarization caused to increase the cross-section of the process. The study of bremsstrahlung due to the scattering of longitudinal polarized electrons by different nuclei was done by Neumcke ${ }^{[11]}$ in 1966 , and after many years, Behncke and $\mathrm{Nakel}^{[12]}$ in1974 calculated the linear polarization of photons in the scattering of electrons with carbon nucleus. In 1989, Caffo and others ${ }^{[13]}$, investigated the polarization of the scattered electrons and of the emitted photons in the bremsstrahlung at high energies. The asymmetry of photon polarization in the bremsstrahlung due to polarized electrons were investigated in 1992 by Mergl and others ${ }^{[14]}$ and in 1996 by Haug ${ }^{[15]}$. In 2002, Likhachev and others ${ }^{[16]}$, calculated experimentally the degree of linear and circular polarization of bremsstrahlung photons, and in 2005, Lee and others ${ }^{[17]}$ investigated the production of bremsstrahlung in the field of heavy atoms at intermediate range of energies. Haug ${ }^{[18]}$ and $\mathrm{Nukel}^{[19]}$, in 2006, both studied the photon polarization and the spin asymmetry in the bremsstrahlung process in details. In 2009, S.A.Alkhateeb ${ }^{[20]}$, obtained the relation between the circular polarization of photons and the longitudinal polarization of the scattered electrons in the electromagnetic field of light nuclei.

\section{II-Formulation of the Problem}

The bremsstrahlung photons $\gamma$ are produced in the process of the interaction of electrons with the field of nuclei:

$e_{i}^{-}+N \rightarrow N+e_{f}^{-}+\gamma$ 
Feynman diagrams of this process are shown in Fig. a.

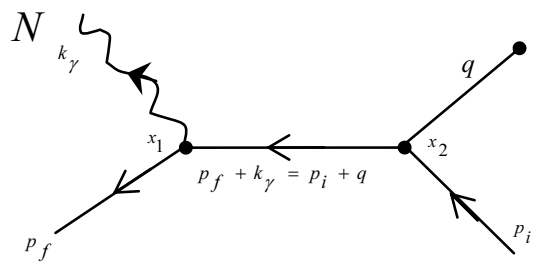

(2)

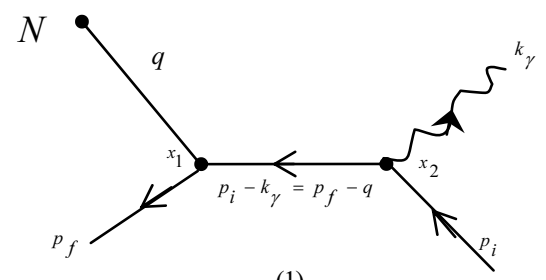

(1)

Fig . a

From these diagrams we see that the momentum transfer to the nucleus in the process is $q=p_{f}+k_{\gamma}-p_{i}$. The matrix element (s-matrix) for the process could be written as the sum of two amplitudes corresponding to the above diagrams (1) and (2) and has the form[3]:

$$
\begin{aligned}
& S_{f i}=e^{2} \int \bar{U}_{f}\left(x_{1}\right) \Gamma\left(x_{1}, x_{2}\right) U_{i}\left(x_{2}\right) d^{4} x_{1} d^{4} x_{2} \\
& \Gamma\left(x_{1}, x_{2}\right)=A_{K}\left(x_{1}, k_{\gamma}\right) G_{f}\left(x_{1}-x_{2}\right) \gamma_{4} A_{N}\left(x_{1}\right)+\gamma_{4} A_{N}\left(x_{2}\right) G_{f}\left(x_{1}-x_{2}\right) A_{K}\left(x_{2}, k_{\gamma}\right)
\end{aligned}
$$

Where $A_{K}$ is the electromagnetic potential of the emitted photon with momentum $k_{\gamma}, A_{N}$ is the potential of the nuclear field, $G_{f}\left(x_{1}-x_{2}\right)$ is the Feynman propagator of the intermediate state between the two points $x_{2}$ and $x_{1}$. The potentials $A_{K}$ and $A_{N}$ are given in the form[5]:

$$
\begin{aligned}
& A_{K}\left(x, k_{\gamma}\right)=\varepsilon^{\mu} \sqrt{\frac{4 \pi}{2 \omega V}}\left(e^{i \bar{k} \cdot \bar{x}}+e^{-i \bar{k} \cdot \bar{x}}\right) \\
& A_{N}(x)=-4 \pi Z e^{2} \int \frac{d^{3} q}{(2 \pi)^{3}} \frac{1}{q^{2}} e^{i \bar{q} \cdot \bar{x}}
\end{aligned}
$$

Where $\varepsilon^{\mu}=(0, \vec{\varepsilon})$ is the polarization vector of the photon, $\omega$ is the photon energy, $\mathrm{V}$ is the normalization volume, $\mathrm{Z}$ is the atomic number of the target nucleus, the potential $A_{N}(x)$ given in (4) represent the coulomb field of nucleus. The differential cross-section of the bremsstrahlung with respect to photon energy is given by [4]:

$$
\frac{d \sigma}{d \omega}=\frac{Z^{2} e^{6} m_{0}^{2}}{\pi^{2}} \frac{\left|p_{f}\right|}{\left|p_{i}\right|} \frac{\omega}{|q|^{4}} F\left(p_{i}, p_{f}, k_{\gamma}\right) d \Omega_{e} d \Omega_{k_{\gamma}}
$$


$\operatorname{In}(6): m_{0}$ is the rest mass of electron, $\mathrm{e}$ is the electronic charge. $d \Omega_{e}$ and $d \Omega_{k_{y}}$ are the solid angles of the scattered electron and the emmited photon respectively, $F\left(p_{i}, p_{f}, k_{\gamma}\right)$ is a function depends on the initial $\left(p_{i}\right)$ and final $\left(p_{f}\right)$ momenta of electron and the photon momentum $\left(k_{\gamma}\right)$. The final form of this function is given by [20]:

$$
\begin{aligned}
F= & \frac{-1}{4 m_{0}^{2}} \frac{1}{\left(p_{i} k_{\gamma}\right)^{2}\left(p_{f} k_{\gamma}\right)^{2}}\left\{4 m_{0}^{2}\left[\left(p_{f} k_{\gamma}\right) E_{f}-\left(p_{i} k_{\gamma}\right) E_{i}\right]^{2}+\left[\left(p_{f} k_{\gamma}\right)^{2}+\left(p_{i} k_{\gamma}\right)^{2}\right] .\right. \\
& {\left.\left[2\left(p_{i} k_{\gamma}\right)\left(p_{f} k_{\gamma}\right)-q^{2} m_{0}^{2}\right]+2\left(p_{i} k_{\gamma}\right) q^{2}\left(p_{f} k_{\gamma}\right)\left[E_{i}^{2}+E_{f}^{2}-\left(p_{i} p_{f}\right)\right]\right\} }
\end{aligned}
$$

here:

$$
\begin{aligned}
& \left(p_{i} k_{\gamma}\right)=\omega\left[E_{i}-p_{i} \cos \theta_{i}\right] \quad, \quad\left(p_{f} k_{\gamma}\right)=\omega\left\lfloor E_{f}-p_{f} \cos \theta_{f}\right\rfloor \\
& q^{2}=-\left[p_{i}^{2}+p_{f}^{2}+\omega^{2}+2 \omega p_{f} \cos \theta_{f}-2 \omega p_{i} \cos \theta_{i}-2 p_{i} p_{f} \cos \left(p_{i}, p_{f}\right)\right] \\
& \cos \left(p_{i}, p_{f}\right)=\cos \theta_{i} \cos \theta_{f}+\sin \theta_{i} \sin \theta_{f} \cos \phi
\end{aligned}
$$

$\theta_{i}, \theta_{f}$ are the polar angles of $p_{i}, p_{f}$ with respect to the photon direction, $\cos \left(p_{i}, p_{f}\right)$ is the angle between $p_{i}$ and $p_{f}$, and $\phi$ is the angle between the planes $\left(p_{i}, k_{\gamma}\right)$ and $\left(p_{f}, k_{\gamma}\right)$.

\section{III-Analytical Results for the Problem}

\section{1- The basic Formula for the Differential Cross-Section}

The differential cross-section of bremsstrahlung in the coulomb field of the nucleus can be obtained from the above relations, and the final form of this cross - section is given by:

$$
\frac{d \sigma}{d \omega}=\eta\left(\frac{1}{q^{4}}\right) F_{0 e}\left(\theta_{i}, \theta_{f}\right) d \Omega_{e} d \Omega_{k_{\gamma}}
$$

Which is known as Bethe - Hietler relation for the bremsstrahlung, where:

$$
\eta=\frac{Z^{2} \alpha^{3}}{(2 \pi)^{2}}\left(\frac{1}{\omega}\right) \frac{p_{f}}{p_{i}}
$$




$$
\begin{aligned}
F_{0 e}\left(\theta_{i}, \theta_{f}\right)= & \frac{p_{i}^{2} \sin ^{2} \theta_{i}}{\Delta_{i}^{2}}\left(4 E_{f}^{2}-q^{2}\right)+\frac{p_{f}^{2} \sin ^{2} \theta_{f}}{\Delta_{f}^{2}}\left(4 E_{i}^{2}-q^{2}\right)+\frac{2 \omega^{2}}{\Delta_{i} \Delta_{f}} . \\
& \left(p_{i}^{2} \sin ^{2} \theta_{i}+p_{f}^{2} \sin ^{2} \theta_{f}\right)- \\
& \frac{2 f\left(\theta_{i}, \theta_{f}\right)}{\Delta_{i} \Delta_{f}}\left(2 E_{i}^{2}-2 E_{f}^{2}-q^{2}\right)
\end{aligned}
$$

$\Delta_{i}=E_{i}-p_{i} \cos \theta_{i} \quad, \quad \Delta_{f}=E_{f}-p_{f} \cos \theta_{f}$

$f\left(\theta_{i}, \theta_{f}\right)=p_{i} p_{f} \sin \theta_{i} \sin \theta_{f} \cos \phi$

$E_{i}, E_{f}$ and $\omega$ are the energies of initial and final electron and of the emitted photon, where:

$$
\omega=E_{i}-E_{f} \quad, \quad E_{i}=\sqrt{p_{i}^{2}+m_{0}^{2}} \quad, \quad E_{f}=\sqrt{p_{f}^{2}+m_{0}^{2}}
$$

\section{2- Study of the Electron and Photon Polarization and the Electro- magnetic Structure of Target Nucleus}

The studying of the interaction between the longitudinally polarized electrons and the electromagnetic field of target nucleus corresponding to the charge distribution $(\mathrm{Ze})$ and the magnetic dipole moment $\left(\mu_{I}\right)$ of the nucleus, taking into consideration the circular polarization of the bremsstrahlung photons, gives the following formula for the differential cross-section of the process:

$$
\begin{aligned}
& d \sigma_{s, s_{e}}\left(\theta_{i}, \theta_{f}, Z e, \mu_{I}\right)=d \sigma_{s, s_{e}}^{e}\left(\theta_{i}, \theta_{f}, Z e\right)+d \sigma_{s, s_{e}}^{m}\left(\theta_{i}, \theta_{f}, \mu_{I}\right) \\
& d \sigma_{s, s_{e}}^{e}\left(\theta_{i}, \theta_{f}, Z e\right)=\frac{1}{2} d \sigma_{0 e}\left(\theta_{i}, \theta_{f}\right)+s_{\gamma} s_{e} d \sigma_{1 e}\left(\theta_{i}, \theta_{f}\right) \\
& d \sigma_{s, s_{e}}^{m}\left(\theta_{i}, \theta_{f}, \mu_{I}\right)=\frac{1}{2} d \sigma_{0 m}\left(\theta_{i}, \theta_{f}\right)+s_{\gamma} s_{e} d \sigma_{1 m}\left(\theta_{i}, \theta_{f}\right)
\end{aligned}
$$

Where $d \sigma_{0 e}=d \sigma_{B H}$ is given by (11) and represent the cross-section due to the charge distribution without studying the polarization of particles and photons.

$$
\begin{aligned}
& d \sigma_{1 e}\left(\theta_{i}, \theta_{f}\right)=\eta\left(\frac{1}{q^{4}}\right) F_{1 e} d \omega d \Omega_{e} d \Omega_{k_{y}} \\
& d \sigma_{0 m}\left(\theta_{i}, \theta_{f}\right)=4 \eta a\left(\mu_{I}\right)\left(\frac{1}{q^{2}}\right) F_{0 m} d \omega d \Omega_{e} d \Omega_{k_{y}}
\end{aligned}
$$


$d \sigma_{1 m}\left(\theta_{i}, \theta_{f}\right)=2 \eta a\left(\mu_{I}\right)\left(\frac{1}{q^{2}}\right) F_{1 m} d \omega d \Omega_{e} d \Omega_{k_{\gamma}}$

Where $I$ is the spin of target nucleon, $d \sigma_{1 e}=d \sigma_{e p}$ is the charged crosssection with studying the polarization, $d \sigma_{0 m}$ the magnetic cross-section without studying polarization, and $d \sigma_{1 m}=d \sigma_{m p}$ is the magnetic crosssection with studying the polarization. The functions $F_{1 e}, F_{0 m}$ and $F_{1 m}$ are given by [20]:

$$
\begin{aligned}
F_{1 e}\left(\theta_{i}, \theta_{f}\right)= & \frac{p_{i}^{2} \sin ^{2} \theta_{i}}{\Delta_{i}^{2}}\left(E_{i} p_{i} \omega \cos \theta_{i}+E_{i} p_{f} \omega \cos \theta_{f}-E_{i} \omega^{2}+m_{0}^{2} \omega\right) \\
+ & \frac{p_{f}^{2} \sin ^{2} \theta_{f}}{\Delta_{f}^{2}}\left(E_{i} p_{i} \omega \cos \theta_{i}+E_{i} p_{f} \omega \cos \theta_{f}+E_{f} \omega^{2}-m_{0}^{2} \omega\right) \\
& -\frac{2 f\left(\theta_{i}, \theta_{f}\right)}{\Delta_{i} \Delta_{f}}\left(E_{i} p_{i} \omega \cos \theta_{i}+E_{i} p_{f} \omega \cos \theta_{f}\right)+m_{0}^{2} \omega f\left(\theta_{i}, \theta_{f}\right)\left(\frac{1}{\Delta_{f}^{2}}-\frac{1}{\Delta_{i}^{2}}\right) \\
+ & \frac{m_{0}^{2} \omega^{2}}{\Delta_{i} \Delta_{f}}\left(p_{f}^{2} \sin ^{2} \theta_{f}-p_{i}^{2} \sin ^{2} \theta_{i}\right)\left(\frac{1}{\omega}+\frac{1}{\Delta_{i}}-\frac{E_{i}}{m_{0}^{2}}\right) \\
F_{0 m}\left(\theta_{i}, \theta_{f}\right)= & \left(q^{2}+4 p_{f}^{2}\right) \frac{p_{i}^{2} \sin ^{2} \theta_{i}}{4 \Delta_{i}^{2}}+\left(q^{2}+4 p_{i}^{2}\right) \frac{p_{f}^{2} \sin ^{2} \theta_{f}}{4 \Delta_{f}^{2}} \\
& -\frac{2 f\left(\theta_{i}, \theta_{f}\right)}{\Delta_{i} \Delta_{f}}\left(\frac{q^{2}}{4}+E_{i} E_{f}-m_{0}^{2}\right)+\frac{\omega^{2}}{2}\left(\frac{q^{2}}{\Delta_{i} \Delta_{f}}+\frac{\Delta_{i}}{\Delta_{f}}+\frac{\Delta_{f}}{\Delta_{i}}+2\right) \\
F_{1 m}\left(\theta_{i}, \theta_{f}\right)= & \left(\frac{\omega}{p}\right)\left(\frac{q^{2}}{4}\left\{2 E_{i}\left(\frac{1}{\Delta_{i}}+\frac{1}{\Delta_{f}}\right)-m_{0}^{2}\left(\frac{1}{\Delta_{i}^{2}}+\frac{1}{\Delta_{f}^{2}}\right)+\frac{2}{\Delta_{i} \Delta_{f}}\left(E_{i}^{2}+E_{i} E_{f}-3 m_{0}^{2}\right)\right\}\right. \\
& \left.-m_{0}^{2}\left(\frac{p_{f}^{2}+2 \omega \Delta_{i}}{\Delta_{i}^{2}}+\frac{p_{i}^{2}-2 \omega \Delta_{f}}{\Delta_{f}^{2}}-\frac{2\left(E_{i} E_{f}-m_{0}^{2}\right)}{\Delta_{i} \Delta_{f}}\right)-\frac{\omega E_{i}}{2}\left(\frac{\Delta_{i}}{\Delta_{f}}-\frac{\Delta_{f}}{\Delta_{i}}\right)\right)
\end{aligned}
$$

The quantity $S_{e}= \pm 1$ represent the helicity of the initial electron. $S_{e}=+1$ for the right-longitudinal electron. $S_{e}=-1$ for the left-longitudinal electron. The quantity $S_{\gamma}= \pm 1$ represent the circular polarization of bremsstrahlung photon. $S_{\gamma}=+1$ for the right circular photon polarization. $S_{\gamma}=-1$ for the left circular photon polarization. 


\section{3- The Angular Distribution of the Circular Polarized Bremsstrahlung Photon}

The integration of (17), (18) and (19) with respect to the solid angle of the scattered electron $d \Omega_{e}$ gives the angular distribution of the circular polarized bremsstrahlung photon into the form:

$$
\begin{aligned}
& d \sigma_{s, s_{e}}\left(\theta_{i}, Z e, \mu_{I}\right)=d \sigma_{s, s_{e}}^{e}\left(\theta_{i}, Z e\right)+d \sigma_{s, s_{e}}^{m}\left(\theta_{i}, \mu_{I}\right) \\
& d \sigma_{s, s_{e}}^{e}\left(\theta_{i}\right)=\frac{1}{2} d \sigma_{o e}\left(\theta_{i}\right)+s_{\gamma} s_{e} d \sigma_{1 e}\left(\theta_{i}\right) \\
& d \sigma_{s, s_{e}}^{m}\left(\theta_{i}\right)=\frac{1}{2} d \sigma_{o m}\left(\theta_{i}\right)+s_{\gamma} s_{e} d \sigma_{1 m}\left(\theta_{i}\right)
\end{aligned}
$$

Where:

$$
\begin{aligned}
& d \sigma_{o e}\left(\theta_{i}\right)=4 \pi \eta \Phi_{0 e}\left(\theta_{i}\right) d \Omega_{k_{\gamma}} \\
& d \sigma_{1 e}\left(\theta_{i}\right)=2 \pi \eta \Phi_{1 e}\left(\theta_{i}\right) d \Omega_{k_{\gamma}} \\
& d \sigma_{o m}\left(\theta_{i}\right)=4 \pi \eta a\left(\mu_{I}\right) \Phi_{0 m}\left(\theta_{i}\right) d \Omega_{k_{\gamma}} \\
& d \sigma_{1 m}\left(\theta_{i}\right)=2 \pi \eta a\left(\mu_{I}\right) \Phi_{1 m}\left(\theta_{i}\right) d \Omega_{k_{\gamma}}
\end{aligned}
$$

In the ultra relatives case $\left(k_{\gamma}, E_{i}, E_{f}>m_{0} c^{2}\right)$, the functions $\Phi_{0 e}, \Phi_{1 e}, \Phi_{0 m}, \Phi_{1 m}$ are given by:

$$
\begin{aligned}
\Phi_{0 e}\left(\theta_{i}\right)= & \frac{1}{8 m_{0}^{2} a^{2} \Delta_{0}}\left\{2(1-\gamma)-\frac{7-2 \gamma}{\Delta_{0}}-\frac{1-\gamma^{2}}{\beta^{2} \Delta_{0}}+\frac{\left(2-2 \gamma+\gamma^{2}\right)\left(2-\Delta_{0}\right)}{\Delta_{0}} \varepsilon_{0}\right. \\
& \left.+\left(\gamma(2-\gamma)+\frac{2\left(2-2 \gamma+\gamma^{2}\right)}{\Delta_{0}}\right) \frac{\varepsilon}{1-\gamma}-\frac{\gamma}{1-\gamma}\left(3+\frac{1-\gamma^{2}}{\beta^{2}}\right) \frac{\varepsilon_{T}}{\beta}\right\} \\
\Phi_{1 e}\left(\theta_{i}\right)= & \frac{1}{8 m_{0}^{2} a^{2} \Delta_{0}}\left\{4 \gamma+\frac{1-6 \gamma}{\Delta_{0}}-\frac{1-\gamma^{2}}{\beta^{2} \Delta_{0}}+\frac{\gamma(2-\gamma)\left(2-\Delta_{0}\right)}{(1-\gamma) \Delta_{0}} L-\frac{\gamma}{1-\gamma}\left(3+\frac{1-\gamma^{2}}{\beta^{2}}\right) \frac{\varepsilon_{0}}{\beta}\right\} \\
\Phi_{0 m}\left(\theta_{i}\right)= & \frac{-3}{2}+\frac{\gamma^{2}\left(1-\gamma-\Delta_{0}\right)}{2 \beta^{2} \Delta_{0}}-\frac{\gamma^{2}}{4(1-\gamma)} \varepsilon+\frac{\gamma^{2}\left(1-\gamma+\gamma \Delta_{0}\right)}{2(1-\gamma) \beta^{3}} \varepsilon_{T}+\frac{\left(2-2 \gamma+\gamma^{2}\right)\left(2+\Delta_{0}\right)}{4(1-\gamma) \Delta_{0}} \varepsilon_{0} \\
\Phi_{1 m}\left(\theta_{i}\right)= & \frac{\gamma}{2}\left\{-1+\frac{\gamma\left(1-\gamma-\Delta_{0}\right)}{\beta^{2} \Delta_{0}}-\frac{\gamma}{2(1-\gamma)} \varepsilon+\frac{\gamma\left(1-\gamma+\gamma \Delta_{0}\right)}{(1-\gamma) \beta^{3}} \varepsilon_{T}+\frac{(2-\gamma)\left(2+\Delta_{0}\right)}{2(1-\gamma) \Delta_{0}} \varepsilon_{0}\right\}
\end{aligned}
$$


where:

$$
\begin{aligned}
& \gamma=\frac{\varepsilon_{\gamma}}{E_{i}}, \quad a=\frac{E_{i}}{m_{0} c^{2}} \quad, \quad \Delta_{0}=1-\cos \theta_{i} \\
& \beta=\sqrt{(1-\gamma)^{2}+2 \gamma \Delta_{0}} \quad, \quad L=2 \ln \frac{2 a(1-\gamma)}{\gamma} \\
& \varepsilon_{0}=2 \ln [2 a(1-\gamma)], \quad \varepsilon_{T}=\ln \frac{\beta-\gamma+1}{\beta+\gamma-1} \quad, \quad \varepsilon=-2 \ln \gamma
\end{aligned}
$$

The second term in (27) and (28) represents the effect of the spin correlation between the electron and photon spins on the charge and magnetic distributions of the target nucleus respectively.

\section{4- Calculation of the Degree of Circular Polarization of the Bremsstrahlung Photons}

The degree of circular polarization in the case of the bremsstrahlung emitted from the collision of electron with nuclei having charge distribution and magnetic dipole moment, is give by:

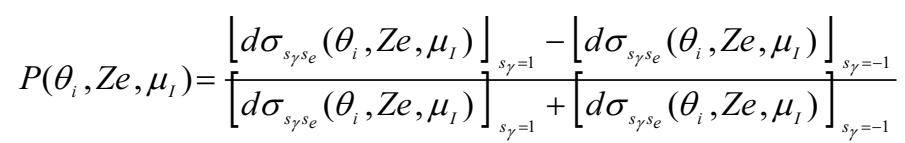

Using the relations (26) - (36) we get the following relation for $(\mathrm{P})$ :

$$
P\left(\theta_{i}, Z e, \mu_{I}\right)=s_{e} p_{e}\left(\theta_{i}\right)\left[\frac{1+a\left(\mu_{I}\right) R_{1}\left(\theta_{i}\right)}{1+a\left(\mu_{I}\right) R_{0}\left(\theta_{i}\right)}\right]
$$

Where: $p_{e}\left(\theta_{i}\right)=\frac{\Phi_{1 e}}{\Phi_{0 e}} \quad, \quad R_{0}\left(\theta_{i}\right)=\frac{\Phi_{0 m}}{\Phi_{0 e}} \quad, \quad R_{1}\left(\theta_{i}\right)=\frac{\Phi_{1 m}}{\Phi_{1 e}}$

$p_{e}\left(\theta_{i}\right)$ represents the degree of charged circular polarization of bremsstrahlung produced in the collision of longitudinally polarized electrons $\left(s_{e}=1,-1\right)$ with the field of nuclei having zero magnetic dipole moment. If we take the averge on the spin state of electron $\left(s_{e}= \pm 1\right)$ and the summation over the photon spin $\left(s_{\gamma}= \pm 1\right)$, we have the relation:

$$
d \sigma\left(\theta_{i}, Z e, \mu_{I}\right)=d \sigma_{0 e}\left(\theta_{i}\right)+d \sigma_{0 m}\left(\theta_{i}\right)=d \sigma_{0 e}\left[1+a\left(\mu_{I}\right) R_{0}\right]
$$

Which represents the angular distribution of the bremsstrahlung produced by un-polarized electron in the field of a nucleus with spin 
I.The second term in (42) gives the magnetic correction to Bethe-Heitler relation for bremsstrahlung. This correction is more effective for the light nuclei, which has a large magnetic moment at large angles of the photon emission.

\section{5- Numerical Application to some Nuclei (Results and Discussion)}

We shall now consider some applications of the obtained formulas to particular nuclei. The electric and magnetic scattering cross-sections for the two nuclei $\left(A l_{13}^{27}\right)$ and $\left(B e_{4}^{9}\right)$ at different values of energy $\varepsilon=(1000,3000,5000 \mathrm{Mev})$ and angles $\theta=\left(10^{\circ}, 30^{\circ}, 60^{\circ}\right)$, and for the polarized and unpolarized cases are considered, and we have the following results:

1- the polarized electric cross-sections for the two nuclei are decreased with increasing both energies and scattering angles, such that $d \sigma_{e}$ is more effective at energy $\varepsilon=1000 \mathrm{Mev}$ and angle $\theta=10^{\circ}$ [Fig.1+3], for ex:

$$
\begin{aligned}
& d \sigma_{e p}=6.7982 \times 10^{-31}\left(\text { at } \varepsilon=1000 \mathrm{Mev}, \theta=10^{\circ}\right), \\
& d \sigma_{e p}=4.72937 \times 10^{-36}\left(\text { at } \varepsilon=5000 \mathrm{Mev}, \theta=60^{\circ}\right)
\end{aligned}
$$

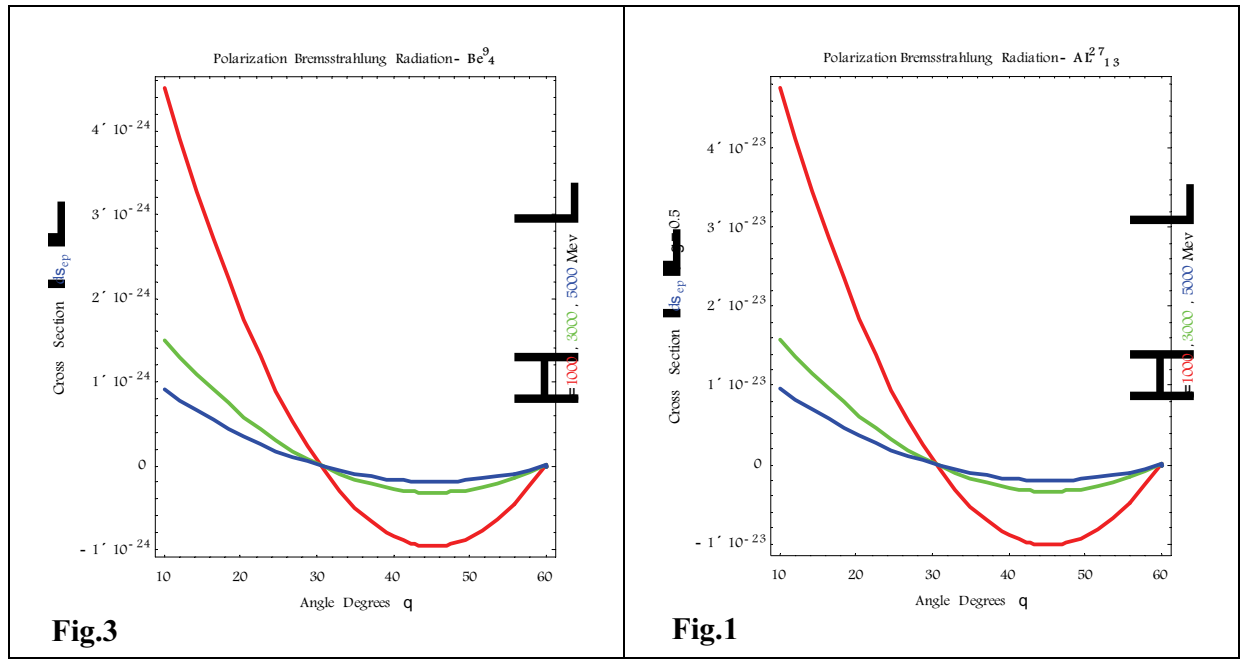

2- the polarized magnetic cross-sections for the two nuclei are also decreased with increasing both energies and scattering angles, such that $d \sigma_{e}$ is more effective at energy $\varepsilon=1000 \mathrm{Mev}$ and angle $\theta=10^{\circ}[\operatorname{Fig}(2)+$ (4)], for ex:

$$
\begin{aligned}
& d \sigma_{m p}=5.7122 \times 10^{-19}\left(\text { at } \varepsilon=1000 \mathrm{Mev}, \theta=10^{\circ}\right), \\
& d \sigma_{m p}=1.05474 \times 10^{-22}\left(\text { at } \varepsilon=5000 \mathrm{Mev}, \theta=60^{\circ}\right)
\end{aligned}
$$




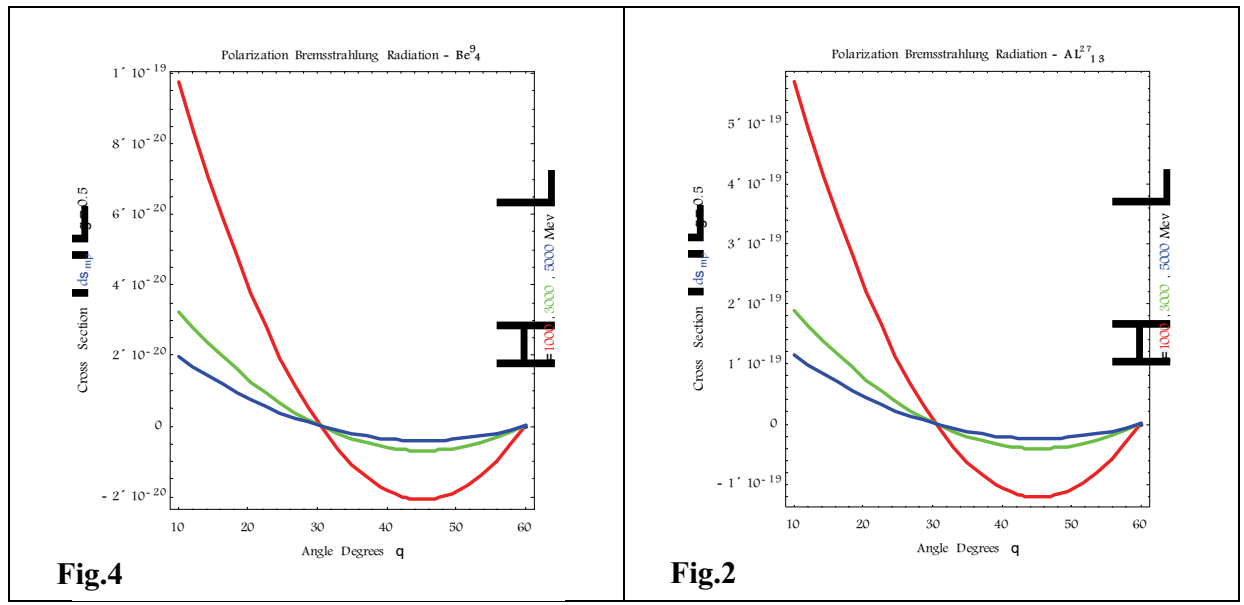

3- comparing the polarized $d \sigma_{e p}$ and unpolarized $d \sigma_{e}$ electric crosssections for the two nuclei $\left(A l_{13}^{27}\right)$ and $\left(B e_{4}^{9}\right)[\operatorname{Fig}(5)+(6)]$, we see that:

i) the values of cross-sections for $\left(A l_{13}^{27}\right)$ are larger than the value for $\left(B e_{4}^{9}\right)$.

ii) the value of polarized $d \sigma_{e p}$ electric cross-section for the two nuclei is larger than the value for the unpolarized $d \sigma_{e}$ cross-section, for ex:

$$
\begin{aligned}
& d \sigma_{e}=6.7982 \times 10^{-31}\left(\text { at } \varepsilon=1000 \mathrm{Mev}, \theta=10^{\circ}\right), \\
& d \sigma_{e p}=4.75822 \times 10^{-23}\left(\text { at } \varepsilon=1000 \mathrm{Mev}, \theta=10^{\circ}\right)
\end{aligned}
$$

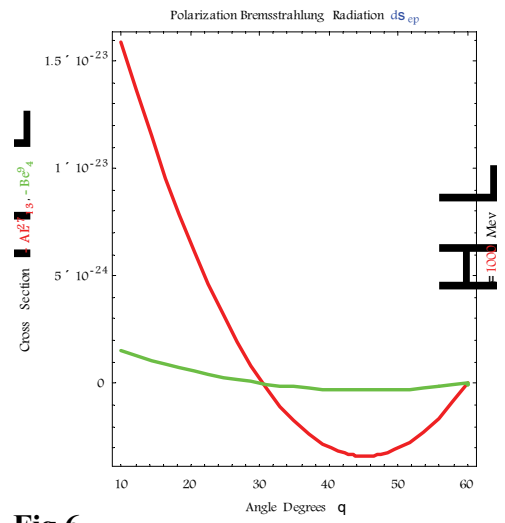

Fig.6

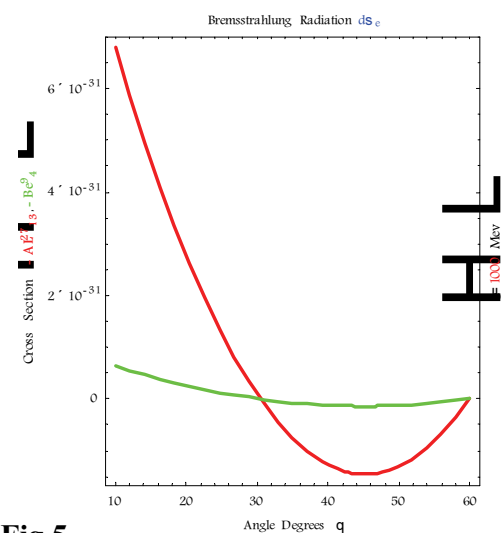

Fig.5 
4- comparing the polarized $d \sigma_{m p}$ and un polarized $d \sigma_{m}$ magnetic cross-sections for the two nuclei $\left(A l_{13}^{27}\right)$ and $\left(B e_{4}^{9}\right)[\operatorname{Fig}(7)+(8)]$,we see that: $\left(B e_{4}^{9}\right)$.

i) the value of cross-sections for $\left(A l_{13}^{27}\right)$ are larger than the value for

ii) the value of polarized $d \sigma_{m p}$ magnetic cross-section for the two nuclei is larger than the value for the un polarized $d \sigma_{m}$ cross-section, for ex:

$$
\begin{aligned}
& d \sigma_{m}=4.73899 \times 10^{-23}\left(\text { at } \varepsilon=1000 \mathrm{Mev}, \theta=10^{\circ}\right), \\
& d \sigma_{m p}=5.7122 \times 10^{-19}\left(\text { at } \varepsilon=1000 \mathrm{Mev}, \theta=10^{\circ}\right)
\end{aligned}
$$

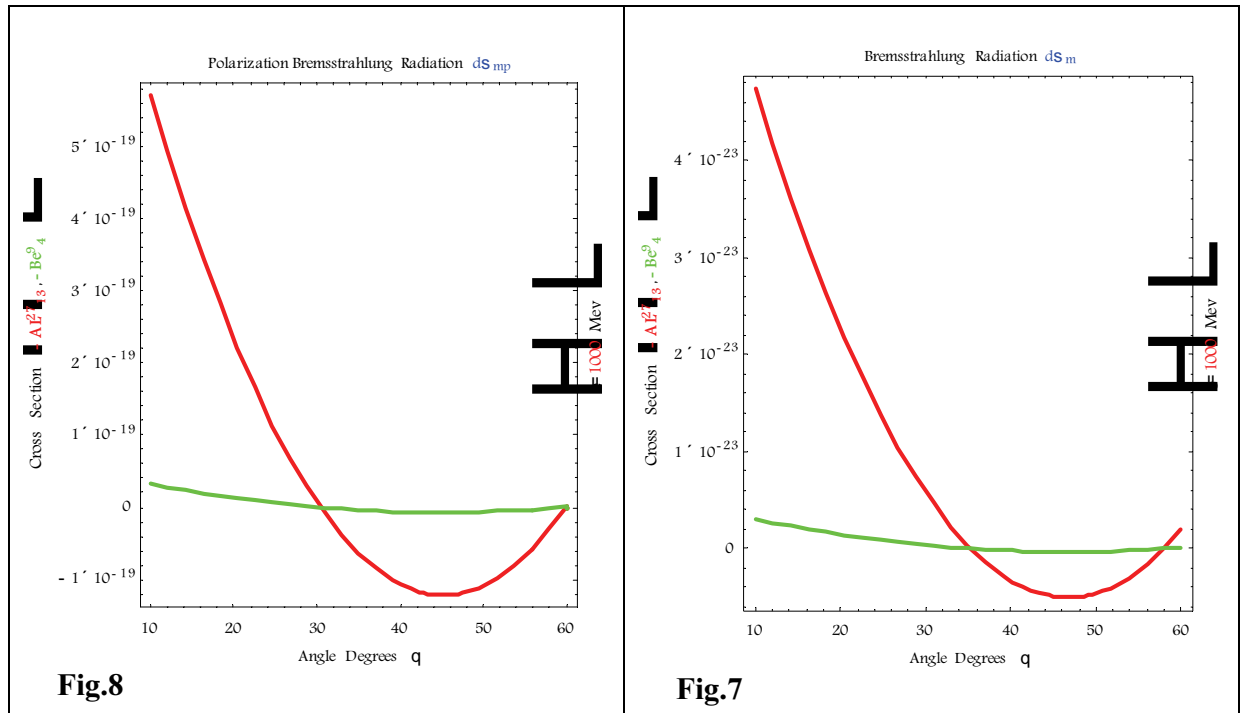

5- comparing the total electric cross-section $d E=d \sigma_{e}+d \sigma_{c p}$ and the total magnetic cross-section $d M=d \sigma_{m}+d \sigma_{m p}$ for the two nuclei [Fig(9) + (10)], we see that:

The values of $d \sigma_{E}$ and $d \sigma_{M}$ for the $\left(A l_{13}^{27}\right)$ nucleus are larger than that for the $\left(B e_{4}^{9}\right)$ nucleus, and have the largest value at $\varepsilon=1000 \mathrm{Mev}, \theta=10^{\circ}$. 


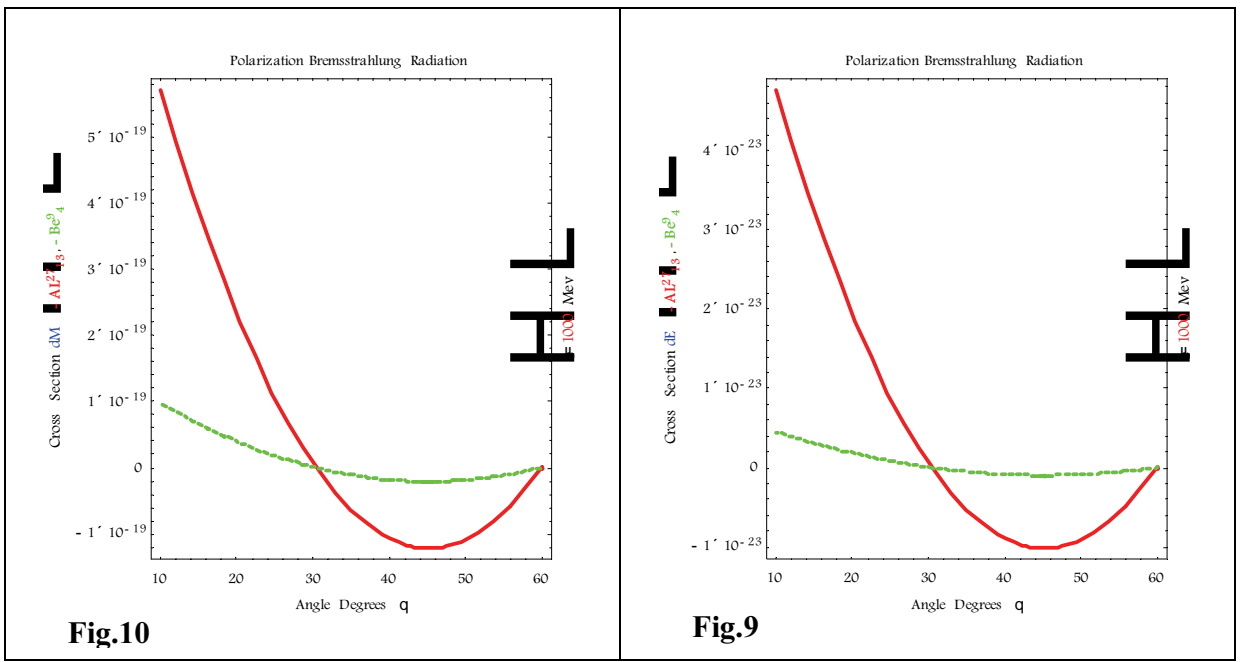

6- comparing between the electric and magnetic parts of the crosssection for the two nuclei [Fig (11) + (12)], where $d E=d \sigma_{e}+d \sigma_{e p}, d M=d \sigma_{m}+d \sigma_{m p}$. We see that:

i) the magnetic part is more effective (i.e has larger values) in the polarized and un polarized cross for the two nuclei.

ii) in the polarized case, the values of cross-sections (electric or magnetic) are larger than the unpolarized case, which means that the study of polarization in the bremsstrahlung process gives us best result, and make the process more evident than that if we neglect the polarization effect, a result in consistent with the experimental results.

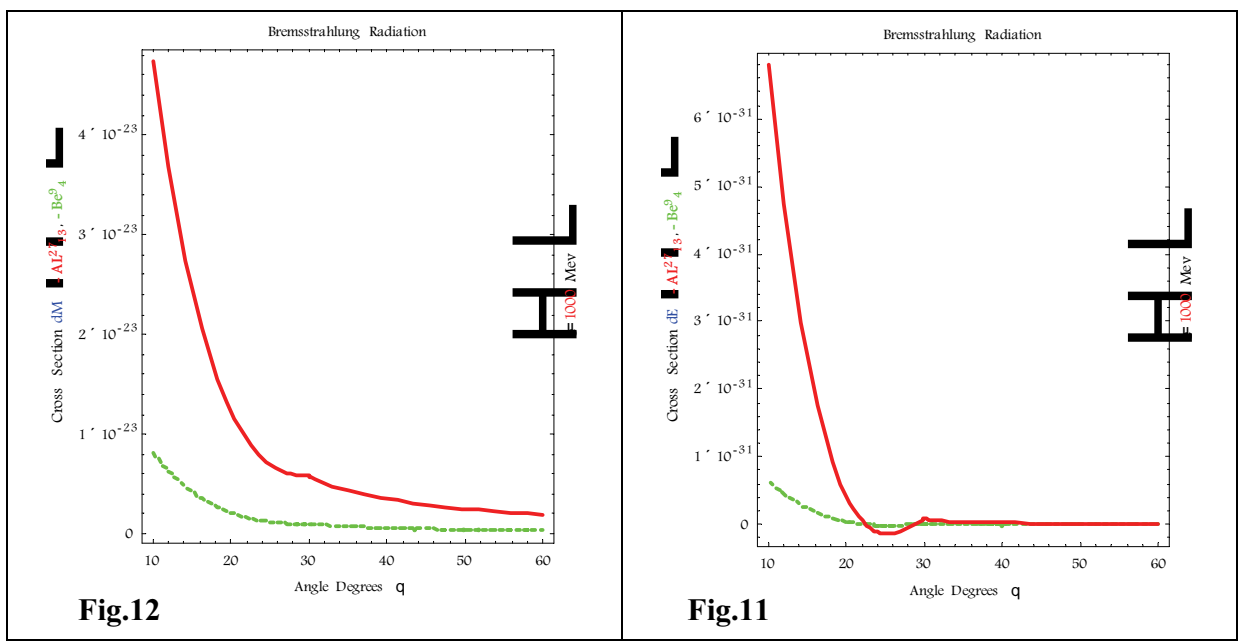




\section{Acknowledgements}

The author would Like to thank the Deanship of Scientific Research at King Abdulaziz University to support this research (no. 21h008/429) and encourage researchers to continue the march of scientific research.

\section{References}

[1] Bethe, H. and Heitler, W. (1934) Proc. Roy. Soc. (London) A146: 83.

[2] Wheeler, J. and Lamb, W. (1939) Phys. Rev. 55: 858.

[3] Bethe, H. and Maximon, L. (1952) Phys. Rev. 87: 156.

[4] Bethe, H. and Maximon, L. (1954) Phys. Rev. 93: 768.

[5] Grodzins, L. (1959) Progr. Nucl. Phys. 7: 163.

[6] Olsen, H. and Maximon, L. (1959) Phys. Rev. 114: 887.

[7] Motz, J. and Placious, R. (1960) Nuovo. Cimento. 15: 572.

[8] Bisi, A. et al. (1962) Nucl. Phys. 36: 320.

[9] Ginsberg, E. and Pratt, R. (1964) Phys. Rev. 134: 773.

[10] Mork, K. and Olsen, H. (1965) Phys. Rev. B140: 1661.

[11] Neumcke, B. (1966) Phys. Lett. 23: 382.

[12] Behncke, H. and Nakel, W. (1974) Phys. Lett. A47: 149.

[13] Caffo, M. et al. (1989) Nucl. Phys. B327: 93.

[14] Mergl, E. et al. (1992) Phys. Rev. Lett. 69: 901.

[15] Haug, E. (1996) Z. Phys. D37: 9.

[16] Likhachev, V. et al. (202) Nucl. Inst. Meth. A495: 139.

[17] Lee, R. et al. (2005) J. Exp. Theor. Phys. 100: 1.

[18] Haug, E. (2006) J. Rad. Phys. and Chem. 75: 1330.

[19] Nakel, W. (2006) J. Rad. Phys. and Chem. 75: 1164.

[20] Alkhateeb, S.A. (2009) Quantum Electrodynamical treatment of leptonic pair production processes, Ph.D. Thesis, K.S.A. 


\section{الاعتماد الاستقطابي للمقاطع المستعرضة للأشعة الكابحة في استقطاب إلكترونات بواسطة نويات ذات عزوم كهرومغنطيسية

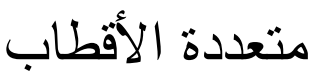

سعدة عبدالله الخطيب

قسم الرياضيات، كلبة العلوم للبنات، جامعة الملك عبدالعزيز جدة، الدملكة العربية السعودية

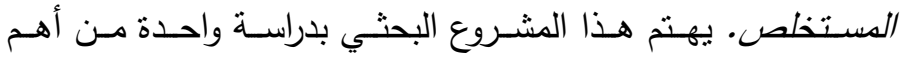
العمليات الإككتروديناميكية الكمية وهي عملية الأشعة الكابحة الناتجة عن التقاعل بين الإكترون ومجال نواة ذات عزوم كهرومغنطيسية متعددة الأقطاب وذللك في حالتين: 1- مــع إهــال الاسـتقطاب الـدائري الفوتـوني والاسـتقطاب الطولي للإلكترون المتفاعل.

r- مع أخذ الاستقطاب الفوتوني والإلكتروني في الاعتبار.

ويثــل البحث استتنتاج العلاقـات الأساسية الخاصـة بعمليـة الأشعة الكابحة في الحالتين واستخدام بعض الطرق العددية للحصول على النتائج الخاصة بالعملية وتحليل تلك النتائج. وبـإجراء مقارنـة بـين الحسالتين المـذكورتين لبيـان مدى تأثير

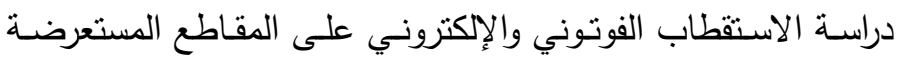

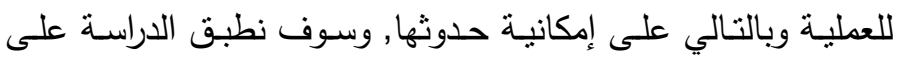
إحدى النويات الخفيفة (Be $)$ وعلى نواة أنقل منها (

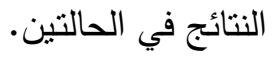

